\title{
p̧ê̂ncia
}

\section{CARACTERIZAÇÃO DA SÍFILIS DIAGNOSTICADA A PARTIR DA TECNOLOGIA TESTE RÁPIDO EM SERVIÇO DE REFERÊNCIA NA CAPITAL TERESINA}

Characterization of syphilis diagnosed from the quick test technology in a reference service in Teresina capital

Caracterización de sífilis diagnosticada de la tecnología de prueba rápida en un servicio de referencia en la capital de Teresina

Karinna Alves Amorim de Sousa - Secretaria de Estado da Saúde do Estado do PiauíSESAPI $\bullet$ Doutora em Enfermagem pela UFPI $\bullet$ Apoiadora do MS no Projeto Sífilis Não!!! Teresina/PI e Timon/MA • E-mail: karinnaduda@gmail.com

Alana Niege Meneses Damasceno • Mestranda em Saúde da Mulher • Coordenadora de IST Aids da Fundação Municipal de Saúde de Teresina-PI •

E-mail: alananiege@yahoo.com.br

Cristiana Portela de Carvalho Rocha $\bullet$ Psicóloga • Coordenadora do Centro de Testagem e Aconselhamento-CTA • E-mail: rochacristiana2015@gmail.com Luciana Sena Sousa $\bullet$ Enfermeira $\bullet$ Mestranda em Saúde da Mulher $\bullet$ Gerente de Atenção à Saúde-SESAPI • E-mail: lucianasenaba@hotmail.com Denis Romulo Leite Furtado • Doutorando em Engenharia Biomédica na Universidade Brasil • Farmacêutico do Laboratório Central-LACEN-PI • E-mail: denisfarmac@gmail.com

Meire Maria de Sousa e Silva • Mestre em Saúde da Família • Técnica da Coordenação de Doenças Transmissíveis da-SESAPI • E-mail: mmssavia@gmail.com Thais Gomes Oliveira dos Reis • Enfermeira do Centro de Testagem e Aconselhamento-CTA • E-mail: cta.pi.the@gmail.com 


\section{RESUMO}

Introdução: A sífilis é uma Infecção Sexualmente Transmissível que atinge mais de 12 milhões de pessoas no mundo e sua eliminação continua a desafiar globalmente os sistemas de saúde. O Brasil, na última década, obteve aumento de notificações de sífilis adquirida. Objetivo: Estimar a prevalência da infecção e caracterizar a população diagnosticada com sífilis a partir de teste rápido realizado no Centro de Testagem e Aconselhamento em Teresina. Metodologia: Trata-se de um estudo descritivo, epidemiológico, de corte transversal realizado por meio dos dados disponíveis no SI-CTA (Sistema de informação do CTA), realizado a partir de levantamento dos registros da população atendida no serviço durante o ano de 2018. Resultados: Foram atendidas 6.664 pessoas que realizaram teste rápido de sífilis, das quais 1.083 tiveram o diagnóstico confirmatório da doença, ocasionando uma prevalência de sífilis foi $16,25 \%$. Sendo $77 \%$ no sexo masculino e $23 \%$ no feminino. Predominância entre pardos autorreferidos (51\%), idade entre 18 a 28 anos (39\%), solteiros (67\%), com escolaridade entre 8 a 11 anos de estudo (56\%). Em relação ao comportamento de risco, 62\% realizava o teste pela primeira vez. Quanto à prática sexual, $59 \%$ afirmaram ter de um a cinco parceiros sexuais no último ano, apenas $6 \%$ relataram sempre usar preservativos, $27 \%$ afirmaram não usar camisinha por confiar no parceiro. O uso de álcool/drogas foi relatado por $57 \%$. A maioria da população atendida foi de homossexuais (52\%), exposição sexual de risco foi relatada por $44 \%$. Conclusões: A prevalência encontrada justifica necessidade de ampliação do acesso ao diagnóstico e tratamento oportuno nas unidades básicas de saúde ou outros serviços com a implantação do teste rápido. O estudo possibilita deduzir a importância do CTA, pois agrega experiência com população mais vulnerável à sífilis, podendo ser referência para serviços na promoção de campanhas educativas e de detecção de casos.

Palavras-Chave: Sífilis. Sorodiagnóstico da sífilis. Epidemiologia.

\section{ABSTRACT}

Introduction: Syphilis is a Sexually Transmitted Infection that affects more than 12 million people worldwide and its elimination continues to challenge health systems globally. Brazil, in the last decade, had an increase in notifications of acquired syphilis. Objective: To estimate the prevalence of infection and to characterize the population diagnosed with syphilis using a rapid test performed at the Testing and Counseling Center in Teresina. Methodology: This is a descriptive, epidemiological, cross-sectional study carried out using data available at SI-CTA (CTA Information System), carried out based on a survey of the records of the population served at the service during 2018. Results: 6,664 people who underwent rapid syphilis test were assisted, of which 1,083 had a confirmatory diagnosis of the disease, causing a prevalence of syphilis was $16.25 \%$. $77 \%$ were male and $23 \%$ female. Predominance among self-reported browns (51\%), aged between 18 to 28 years (39\%), single $(67 \%)$, with schooling between 8 to 11 years of study (56\%). Regarding risk behavior, $62 \%$ performed the test for the first time. As for sexual practice, 59\% said they had one to five sexual partners in the last year, only $6 \%$ reported always using condoms, $27 \%$ 
said they did not use condoms because they trusted their partner. Alcohol / drug use was reported by $57 \%$. The majority of the population served was homosexual (52\%), sexual risk exposure was reported by $44 \%$. Conclusions: The prevalence found justifies the need to expand access to diagnosis and timely treatment in basic health units or other services with the implementation of the rapid test. The study makes it possible to deduce the importance of CTA, as it adds experience to the population most vulnerable to syphilis, and can be a reference for services in the promotion of educational campaigns and case detection.

Keywords: Syphilis. Serodiagnosis of syphilis. Epidemiology

\section{RESUMEN}

Introducción: La sífilis es una infección de transmisión sexual que afecta a más de 12 millones de personas en todo el mundo y su eliminación continúa desafiando los sistemas de salud a nivel mundial. Brasil, en la última década, tuvo un aumento en las notificaciones de sífilis adquirida. Objetivo: Estimar la prevalencia de infección y caracterizar a la población diagnosticada con sífilis utilizando una prueba rápida realizada en el Centro de Pruebas y Asesoramiento en Teresina. Metodología: Este es un estudio descriptivo, epidemiológico, transversal realizado con los datos disponibles en SI-CTA (Sistema de Información de CTA), realizado en base a una encuesta de los registros de la población atendida en el servicio durante 2018. Resultados: 6.664 personas que se sometieron a la prueba rápida de sífilis fueron asistidas, de las cuales 1.083 tuvieron un diagnóstico confirmatorio de la enfermedad, causando una prevalencia de sífilis del $16.25 \% .77 \%$ eran hombres y $23 \%$ mujeres. Predominio entre marrones autoinformados (51\%), con edades comprendidas entre 18 y 28 años (39\%), solteros (67\%), con escolaridad entre 8 y 11 años de estudio (56\%). Con respecto al comportamiento de riesgo, el $62 \%$ realizó la prueba por primera vez. En cuanto a la práctica sexual, el 59\% dijo que tenía de una a cinco parejas sexuales en el último año, solo el 6\% informó que siempre usaba condones, el $27 \%$ dijo que no los usaba porque confiaban en su pareja. El consumo de alcohol / drogas se informó en un 57\%. La mayoría de la población atendida era homosexual (52\%), la exposición al riesgo sexual se informó en un $44 \%$. Conclusiones: La prevalencia encontrada justifica la necesidad de ampliar el acceso al diagnóstico y al tratamiento oportuno en las unidades básicas de salud u otros servicios con la implementación de la prueba rápida. El estudio permite deducir la importancia de la CTA, ya que agrega experiencia a la población más vulnerable a la sífilis, y puede ser una referencia para los servicios en la promoción de campañas educativas y la detección de casos.

Palabras clave: Sífilis. Serodiagnóstico de sífilis. Epidemiología. 


\section{Introdução}

A sífilis é uma Infecção Sexualmente Transmissível (IST) milenar e persistente. Caracteriza-se como uma doença infecciosa sistêmica de evolução crônica causada pela bactéria Treponema pallidum, com transmissão predominantemente sexual. Fatores relevantes na transmissibilidade da sífilis podem estar relacionados a aspectos sociais, biológicos, culturais e comportamentais que influenciam a ocorrência da doença na população ${ }^{1}$.

Segundo a Organização Mundial da Saúde (OMS), atinge mais de 12 milhões de pessoas em todo o mundo e sua eliminação continua a desafiar globalmente os sistemas de saúde com cerca de 6 milhões de novos casos a cada ano. Durante o período gestacional, a sífilis leva a mais de 300.000 mortes fetais e neonatais por ano no mundo e aumenta o risco de morte prematura em outras 215.000 crianças $^{2}$.

No Brasil, a sífilis foi declarada como um grave problema de saúde pública, na última década observou-se aumento de notificações de casos de sífilis adquirida, sífilis em gestantes e sífilis congênita, que pode ser atribuído, em parte, ao aprimoramento do sistema de vigilância e à ampliação da utilização de testes rápidos. Em 2017, foram notificados 119.800 casos de sífilis adquirida (taxa de detecção de 58,1 casos/100 mil habitantes); 49.013 casos de sífilis em gestantes (taxa de detecção de 17,2/1.000 nascidos vivos); 24.666 casos de sífilis congênita (taxa de incidência de 8,6/1.000 nascidos vivos); e 206 óbitos por sífilis congênita (taxa de mortalidade de 7,2/100 mil nascidos vivos). Em comparação ao ano de 2016, observou-se aumento de $28,5 \%$ na taxa de detecção em gestantes, $16,4 \%$ na incidência de sífilis congênita e 31,8\% na incidência de sífilis adquirida ${ }^{3}$.

A testagem para detecção da sífilis é uma das estratégias para possibilitar diagnóstico precoce e tratamento oportuno. É reconhecida como uma medida custoefetiva. A utilização de testes com resultado imediato, tem sido indicada em situações de baixo acesso a testes de laboratórios. Os testes rápidos (TR) treponêmicos apresentam sensibilidade e especificidade semelhantes aos testes treponêmicos realizados em laboratório. Sua sensibilidade e especificidade são comparáveis às de testes não treponêmicos ${ }^{4}$. 
O efetivo controle da sífilis depende, em grande medida, da disposição e vontade política de gestores para colocar em prática um movimento em prol da qualidade da atenção à saúde, promover mobilização nacional para ampliação do acesso ao diagnóstico da população geral e das populações-chave e estabelecer parcerias de base comunitária, além de vencer obstáculos quanto à administração da medicação benzilpenicilina benzatina para tratamento da sífilis na Atenção Básica ${ }^{5}$.

Atualmente, a oferta de teste rápido de sífilis é crescente, mas sua utilização e cobertura na atenção primária ainda não são satisfatórias. Assim, o serviço Centro de Testagem e Aconselhamento - CTA caracteriza-se estrategicamente como uma das respostas à epidemia das Infecções sexualmente transmissíveis - IST, e assume papel de referência no campo da prevenção, especialmente para o acesso universal à testagem e aconselhamento da população geral e os segmentos populacionais considerados em situação de maior risco e vulnerabilidade ${ }^{6-7}$.

Dentre as ações desenvolvidas pelo CTA destacam-se: o aconselhamento em IST nos momentos pré e pós-teste, oferta de testagem para triagem e diagnóstico do HIV, Sífilis e Hepatites B e C, palestras, oficinas e disponibilização dos insumos de prevenção (camisinha masculina, feminina, gel lubrificante) e alimentação do sistema de informação do serviço - SI-CTA. A organização dos processos de trabalho no CTA deve estar centrada em cada usuário e em suas necessidades, é necessário entender a especificidade de cada indivíduo, de forma a ajudá-los a melhor avaliar suas situações de vulnerabilidade e a escolher conscientemente a estratégia de prevenção mais adequada dentre as tecnologias existentes ${ }^{8}$.

Esse estudo tem como objetivos: Estimar a prevalência da infecção por sífilis e caracterizar a população diagnosticada a partir de teste rápido realizado no CTA em Teresina durante o ano 2018.

\section{Metodologia}

Trata-se de um estudo descritivo, epidemiológico, de corte transversal realizado por meio dos dados disponíveis no SI-CTA (Sistema de informação do 
CTA), realizado no mês de março de 2019 a partir de levantamento dos registros da população atendida no serviço durante o ano de 2018.

O CTA é um serviço localizado em Teresina, de gestão estadual, por demanda espontânea que atende ao público oriundo da capital, interior e de outros estados, funciona diariamente sendo referência na prevenção e diagnóstico das IST, que oferta o teste rápido de sífilis treponêmico como triagem inicial e aos casos reagentes, procede com o exame não treponêmico VDRL, para conclusão do diagnóstico, associado à clínica e epidemiologia, confirmando o diagnóstico realizase notificação tratamento e seguimento clínico. Aos casos não reagentes no teste rápido, é discutido sobre janela imunológica, importância da adoção de comportamento seguro. E disponibiliza-se preservativos masculino, feminino, gel lubrificante e cartilha informativa.

Foram incluídos no estudo os casos confirmados com a doença, provenientes de resultados do teste rápido reagente, seguidamente do VDRL positivo, considerando as situações pertinentes para critérios de definição de casos de sífilis e sendo maiores de idade.

Os dados foram digitados e analisados com a utilização do aplicativo Microsoft Excel 2016. Para determinar a prevalência da sífilis e os fatores de risco, foram utilizadas estatísticas descritivas simples. A pesquisa obedeceu todos os aspectos éticos, garantida a confidencialidade, a privacidade, a não estigmatização e a não utilização de informações em prejuízo das pessoas, conforme os princípios norteadores dispostos na Resolução no 466/12, do Conselho Nacional de Saúde, que aprova diretrizes e normas regulamentadoras de pesquisas envolvendo seres humanos.

\section{Resultados}

Durante o ano avaliado, foram atendidas 6.664 pessoas que realizaram teste rápido de sífilis, das quais 1.083 tiveram o diagnóstico confirmatório da doença, ocasionando uma prevalência de sífilis foi $16,25 \%$, desses, sendo $77 \%$ no sexo masculino e $23 \%$ no feminino. Observou-se predominância entre pardos 
autorreferidos (51\%), idade entre 18 a 28 anos (39\%), solteiros (67\%), com escolaridade entre 8 a 11 anos de estudo (56\%) (Tabela 1).

Tabela 01: Características sócio demográficas da população em estudo, relacionadas à Sífilis. (n=1.083). Teresina-Piauí, 2019

\begin{tabular}{|c|c|c|}
\hline \multirow{2}{*}{ Variáveis } & \multicolumn{2}{|c|}{ Sífilis } \\
\hline & $\mathbf{n}$ & $\%$ \\
\hline \multicolumn{3}{|l|}{ Sexo } \\
\hline Masculino & 834 & 77 \\
\hline Feminino & 249 & 23 \\
\hline \multicolumn{3}{|l|}{ Cor } \\
\hline Branco & 282 & 26 \\
\hline Pardo & 553 & 51 \\
\hline Preto & 248 & 23 \\
\hline \multicolumn{3}{|l|}{ Situação Conjugal } \\
\hline Solteiro/separado/Viúvo & 726 & 67 \\
\hline Casado/amigado/união estável & 357 & 33 \\
\hline \multicolumn{3}{|l|}{ Idade (anos) } \\
\hline 18 a 28 & 422 & 39 \\
\hline 29 a 39 & 401 & 37 \\
\hline 40 a 50 & 108 & 10 \\
\hline 51 a 61 & 87 & 8 \\
\hline$\geq 62$ & 65 & 6 \\
\hline \multicolumn{3}{|l|}{ Escolaridade (anos de estudo) } \\
\hline Nenhuma & 22 & 2 \\
\hline$<4$ & 54 & 5 \\
\hline 4 a 7 & 271 & 25 \\
\hline 8 a 11 & 606 & 56 \\
\hline$>11$ & 130 & 12 \\
\hline
\end{tabular}

Fonte: SI CTA Teresina - PI

A Tabela 02 apresenta a distribuição dos participantes em relação ao comportamento de risco para sífilis, $62 \%$ realizava o teste pela primeira vez. Quanto à prática sexual, 59\% afirmaram ter de um a cinco parceiros sexuais no último ano, apenas 6\% relataram sempre usar preservativos nas relações sexuais, 27\% afirmaram não usar camisinha por confiar no parceiro. O uso sempre ou às vezes de álcool e/ou outras drogas previamente as relações sexuais foi relatado por $57 \%$. 
Tabela 02: Características comportamentais da população em estudo, relacionadas exposição de risco para Sífilis. (n=1083). Teresina-Piauí, 2019

\begin{tabular}{|c|c|c|}
\hline \multirow{2}{*}{ Variáveis } & \multicolumn{2}{|c|}{ Sífilis } \\
\hline & $\mathbf{N}$ & $\%$ \\
\hline \multicolumn{3}{|l|}{ Já realizou teste para sífilis anteriormente } \\
\hline Sim & 412 & 38 \\
\hline Não & 671 & 62 \\
\hline \multicolumn{3}{|l|}{$N^{o}$ de parceiros sexuais nos últimos 12 meses } \\
\hline Nenhum & 13 & 1 \\
\hline 1 a 5 & 641 & 59 \\
\hline 6 a 10 & 353 & 32 \\
\hline$>11$ & 76 & 8 \\
\hline \multicolumn{3}{|l|}{$\begin{array}{l}\text { Tem hábito de usar álcool/drogas antes das } \\
\text { relações sexuais }\end{array}$} \\
\hline Não usa álcool nem drogas ou não fez sexo & 260 & 24 \\
\hline Usa álcool sempre ou as vezes & 617 & 57 \\
\hline Usa outras drogas sempre ou as vezes & 206 & 19 \\
\hline \multicolumn{3}{|l|}{ Costuma usar camisinha nas relações sexuais } \\
\hline Sempre usa & 65 & 6 \\
\hline Não usa ou não fez sexo & 487 & 45 \\
\hline As vezes usa & 531 & 49 \\
\hline \multicolumn{3}{|l|}{$\begin{array}{l}\text { Motivo de usar as vezes ou não usar } \\
\text { camisinha }\end{array}$} \\
\hline Não gosta & 163 & 16 \\
\hline Não dispunha no momento & 254 & 25 \\
\hline Confia no parceiro & 275 & 27 \\
\hline Sob efeito de álcool/drogas & 132 & 13 \\
\hline Não deu tempo/tesão & 194 & 19 \\
\hline
\end{tabular}

Fonte: SI CTA Teresina - PI

Com relação ao tipo de população atendida pelo CTA, observou-se predominância de homossexuais (52\%), a exposição sexual de risco foi relatada como motivo de procura pelo serviço por $44 \%$, maioria dos usuários (39\%) souberam do CTA por meio da indicação de amigos e outros usuários (Tabela 3). 
Tabela 03: Distribuição do recorte populacional do estudo e a procura pelo serviço. ( $\mathrm{n}=1083)$. Teresina-Piauí, 2019

\begin{tabular}{lcc}
\hline \multicolumn{1}{c}{ Variáveis } & Sífilis \\
\cline { 2 - 3 } & N & \% \\
\hline Categoria do usuário* & 32 & 3 \\
População confinada & 54 & 5 \\
Profissional do sexo & 159 & 15 \\
Usuário de álcool/drogas & 466 & 43 \\
Heterossexual & 563 & 52 \\
Homossexual(Gay/HSH, lésbica, travesti, & 54 & 5 \\
trans) & 43 & 4 \\
Bissexual & 141 & 13 \\
Situação de rua & 17 & 2 \\
Estudante & & \\
Gestante & 141 & 13 \\
Motivo da procura pelo serviço CTA & 159 & 15 \\
Encaminhado de outros serviços de saúde & 218 & 20 \\
Encaminhado de clínicas de recuperação & 87 & 8 \\
Saber se tem doença & 478 & 44 \\
Apresenta sinais/sintomas de IST & & \\
Teve exposição sexual de risco & 98 & 9 \\
Origem da clientela/como soube do serviço & 422 & 39 \\
Material informativo/campanhas & 87 & 8 \\
Amigos ou indicação de outros usuários & 173 & 16 \\
Jornal, rádio, televisão, internet & 303 & 28 \\
Profissionais de saúde ou da educação & & \\
Movimentos sociais, ONG's &
\end{tabular}

Fonte: SI CTA Teresina - PI *múltipla escolha

\section{Discussão}

Este estudo propôs descrever o perfil epidemiológico, comportamentos de risco e a frequencia de sífilis na população usuária do serviço CTA no município de Teresina, tendo encontrado alta prevalência de sífilis (16,25\%). Estudos nacionais realizados com população específica, em situação de rua, encontrou resultados semelhantes, com prevalência de 5,7\% e 7,0\% em São Paulo9-10.

A sífilis permanece como um importante problema de saúde pública, apesar de possuir métodos diagnósticos e tratamento simples. O TR para sífilis tem sido apontado pela OMS, como ferramenta para diagnóstico rápido, a ser utilizado em situações específicas, onde exista dificuldade de acesso a serviços ou a insumos 
laboratoriais ${ }^{11}$. Nesta pesquisa observou-se que mais da metade dos diagnosticados com sífilis faziam o teste para sífilis pela primeira vez, denotando a insuficiência do acesso ao diagnostico bem como a não percepção dos profissionais de saúde implementar solicitação da testagem como rotina às pessoas com vida sexual ativa.

Além do diagnóstico precoce, é primordial a oferta do tratamento adequado para quebra da cadeia de transmissão e controle da doença. A penicilina benzatina foi reconhecida pela 69ª Assembleia Mundial da Saúde, em maio de 2016, como um medicamento essencial para controle da transmissão vertical de sífilis, e globalmente tem apresentado escassez há alguns anos. No Brasil, assim como em outros países, desde 2014 enfrenta-se o desabastecimento de penicilina benzatina, devido à falta mundial de matéria prima para a sua produção. Além do desabastecimento, dados do segundo ciclo do Programa Nacional de Melhoria do Acesso e da Qualidade da Atenção Básica (PMAQ-AB), em 2013/2014, indicam que apenas 55\% das equipes de saúde da família, aplicam penicilina benzatina na Atenção Básica, o que pode contribuir para o aumento dos casos de sífilis ${ }^{2}$.

Observa-se que o CTA é mais procurado por populações específicas que vivenciam situações de maior vulnerabilidade, apenas $2 \%$ de gestantes buscaram realizar exames no serviço, dessa forma, prevaleceu o diagnóstico da sífilis adquirida, que vem também se instalando entre os segmentos mais jovens da população, sobretudo entre homens, neste estudo representado por $77 \%$, o que impõe a necessidade de desenvolver estratégias intersetoriais, incluindo ações de prevenção nas escolas e nas redes de interação juvenil. Esse movimento contribui para a promoção da saúde integral do homem, considerando que a maioria destes só procura serviços de saúde quando doentes. Uma cultura que precisa ser revertida, principalmente em se tratando de IST.

Outro fator importante, inclusive pelo fato da infecção estar presente na população feminina e de idade fértil, é o diagnóstico precoce e tratamento adequado às gestantes, no intuito de se prevenir as graves consequências da sífilis congênita, sendo os melhores resultados obtidos quando o tratamento é efetuado por volta da $24^{\circ}$ a $28^{\circ}$ semana gestacional. O tratamento adequado com penicilina é capaz de prevenir $97 \%$ dos casos de transmissão vertical ${ }^{12-13}$. 
Importante ressalvar no ponto de vista de saúde pública, evidenciado nesta pesquisa, a alta proporção de pessoas que relataram fazer uso de álcool e/ou outras drogas previamente às relações sexuais, tendo em vista comprovação do poder que estas substancias exercem de rebaixar nível de consciência influenciando diretamente para não adoção de comportamento seguro diante das exposições de risco. Considerando o impacto negativo deste fato na saúde da população, seria desejável a realização de ações de redução de danos associadas às intervenções de prevenção e de cuidado integral à saúde.

Chama atenção a baixa adesão ao uso de preservativos nas relações sexuais. Sabe-se que sífilis é um marcador de sexo desprotegido ${ }^{10}$. Dessa forma, seria interessante buscar estratégias para melhoria do acesso e adesão aos preservativos, através de dispensação facilitada em locais públicos de grande circulação de pessoas, bem como mecanismos por meio da educação em saúde, tornar a camisinha mais atrativa ao público adulto jovem.

Torna-se necessário superar as falhas na implementação das medidas de controle da sífilis, sendo fundamental a busca de estratégias diferenciadas para alcance dos públicos socialmente mais vulneráveis, tendo em vista este estudo revelou alta prevalência numa população específica, que são os mais afetados pela infecção da sífilis, pelo contexto socioeconômico e cultural ${ }^{14}$.

\section{Conclusões}

A prevalência encontrada justifica necessidade de intensificar ações que possibilitem ampliação do acesso ao diagnóstico e tratamento oportuno para controle da infecção. O estudo possibilita deduzir a importância de serviços específicos como o CTA, que agrega experiência com população mais vulnerável à sífilis, podendo ser referência para outros serviços na promoção de campanhas educativas e de detecção de casos. Também torna-se necessário adoção de estratégias de tornar mais evidente a informação para a população sobre o aumento dos casos e formas de prevenção da doença. 


\section{Referências}

1. Kent ME, Romanelli F. Reexamining Syphilis: an update on epidemiology, Clinical Manifestations, and Management. Ann Pharmacother. 2008;42(2):226-36.

2. Unemo M, Bradshaw CS, Hocking JS et al. Sexually transmitted infections: challenges ahead. Lancet Infect. Dis. 2017(8):e235-e2792017.

3. Brasil. Boletim Epidemiológico. Secretaria de Vigilância em Saúde. Ministério da Saúde. Brasil, 2018;49(45).

4. Brasil. Protocolo Clínico e Diretrizes Terapêuticas para Profilaxia Antirretroviral Pós-Exposição de Risco à Infecção pelo HIV. Departamento de DST, Aids e Hepatites Virais. Brasília: Ministério da Saúde; 2017.

5. Monteiro SS. et al. Discursos sobre sexualidade em um Centro de Testagem e Aconselhamento (CTA). Ciênc. saúde coletiva. 2014 jan 19(1)137-46.

6. Kahn JG, Jiwani A, Gomez GB, Hawkes SJ, Chesson HW, Broutet N, et al. The cost and cost-effective - ness of scaling up screening and treatment of syphilis in pregnancy: a model. PLoS One. 2014;9(1):e87510.

7. Myer L, Wilkinson D, Lombard C, Zuma K, Rotch - ford K, Karim SS. Impact of on site testing for maternal syphilis on treatment delays, treatment rates, and perinatal mortality in rural South Africa: a randomised controlled trial. Sex Transm Infect. 2003;79(3):208-13.

8. Tucker JD, Bu J, Brown LB, Yin YP, Chen XS, Cohen MS. Accelerating worldwide syphilis screening through rapid testing: a systematic review. Lancet Infect Dis. 2010;10(6):381-6.

9. Brito VOC, Parra D, Facchini R, Buchalla CM. HIV infection, hepatitis B and C and syphilis in homeless people, in the city of São Paulo, Brazil. Rev Saúde Pública. 2007;41(Suppl 2):47-56.

10. Pinto VM, Tancredi MV, Alencae HDR, Camolesi E, Holcman MM, Grecco JP, et al. Prevalência de Sífilis e fatores associados a população em situação de rua de São Paulo, Brasil, com utilização de Teste Rápido. Rev bras epidemiol. 2014;17(2):341354.

11. World Health Organization. Special Programme for Research and Training in Tropical Diseases (WHO/TDR). Laboratory-based evaluation of rapid syphilis diagnostics. Sexually Transmitted Diseases Diagnostics Initiative (SDI). 2003.

12. Berman SM. Maternal syphilis: pathophysiology and treatment. Bull World Health Organ. 2004;82(6):433-8. 
13. Blencowe $H$, Cousens $S$, Kamb M, Berman S, Lawn JE. Lives saved tool supplement detection and treatment of syphilis in pregnancy to reduce syphilis related stillbirths and neonatal mortality. BMC Public Health. 2011;11(suppl 3):S9.

14. Domingues RMSM, Leal MC. Incidência de sífilis congênita e fatores associados à transmissão vertical da sífilis: dados do estudo Nascer no Brasil. Cad Saúde Pública. 2016;32(6):e00082415. 